\title{
How Do Sketching and Non-Sketching Actions Convey Design Intent?
}

\author{
Senthil Chandrasegaran \\ College of Engineering \\ University of California, Davis \\ schandrasegaran@ucdavis.edu
}

\author{
Devarajan Ramanujan \\ Department of Engineering \\ Aarhus University, Denmark \\ devr@eng.au.dk
}

\author{
Niklas Elmqvist \\ College of Information Studies \\ Department of Computer Science \\ University of Maryland, College Park \\ elm@umd.edu
}
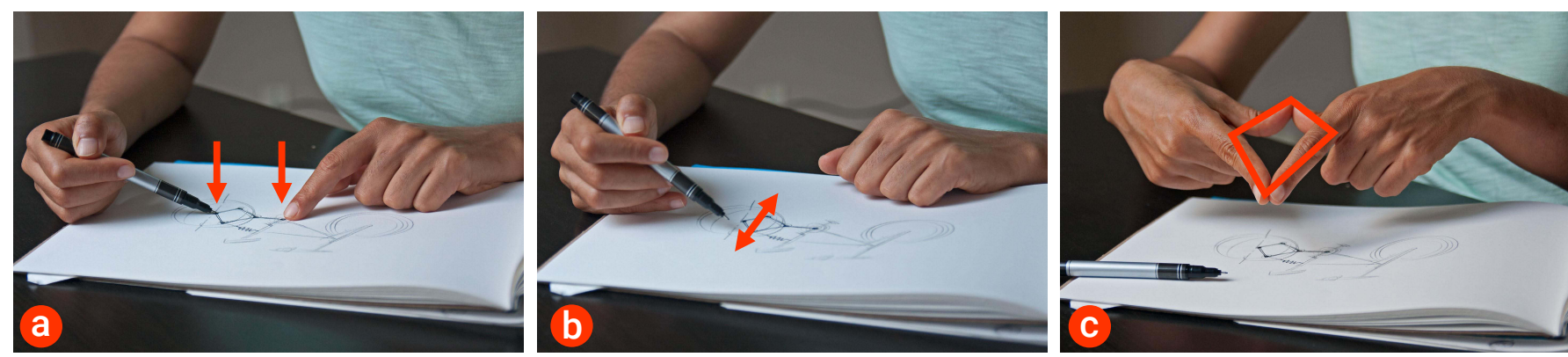

Figure 1. Designers use more than sketches and annotations to express intent. Our observational study revealed three main ways (along with speech) in which designers express intent: (a) on-the-surface "pointing" actions to indicate parts of a design, (b) above-the-surface gestures with fingers or pencils to show movement, shape, or area, and (c) away-from-the-surface gestures, using their hands or other objects as proxies for components or assemblies.

\begin{abstract}
Sketches are much more than marks on paper; they play a key role for designers both in ideation and problem-solving as well as in communication with other designers. Thus, the act of sketching is often enriched with annotations, references, and physical actions, such as gestures or speech-all of which constitute meta-data about the designer's reasoning. Conventional paper-based design notebooks cannot capture this rich meta-data, but digital design notebooks can. To understand what data to capture, we conducted an observational study of design practitioners where they individually explore design solutions for a set of problems. We identified sketching and non-sketching actions that reflect their exploration of the design space. We then categorized the captured meta-data and mapped observed physical actions to design intent. These findings inform the creation of future digital design notebooks that can better capture designers' reasoning during sketching.
\end{abstract}

\section{Author Keywords}

Sketching; ideation; observational study.

\section{ACM Classification Keywords}

H.5.2 Information Interfaces and Presentation: User Interfaces-Interaction styles

Permission to make digital or hard copies of all or part of this work for personal or classroom use is granted without fee provided that copies are not made or distributed for profit or commercial advantage and that copies bear this notice and the full citation on the first page. Copyrights for components of this work owned by others than ACM must be honored. Abstracting with credit is permitted. To copy otherwise, or republish, to post on servers or to redistribute to lists, requires prior specific permission and/or a fee. Request permissions from permissions@acm.org.

DIS '18, June 9-13, 2018, , Hong Kong

(C) 2018 ACM. ISBN 978-1-4503-5198-0/18/06 . \$ \$15.00

DOI: https://doi .org/10.1145/3196709. 3196723

\section{INTRODUCTION}

Design is not just a mental process for the designer: it also involves physical actions, such as drawing depictions on paper, making movements of a pencil or hands without creating physical depictions, and looking at existing depictions to interpret them [38]. These physical actions give insight into the designer's thought process, and help other collaborators (and sometimes the designers themselves) understand the rationale behind their decisions. The design notebook is typically the designer's medium of choice for recording ideas. However, paper notebooks can only capture actions that mark the paper, i.e. the depictions themselves. With the recent proliferation of handheld computing devices, there is a rising trend in digital applications to replace the design notebook, but these tend to still mostly emulate paper without fully transcending the medium to also capture the rationale behind the pen marks.

Hand-held personal computing devices such as smartphones and tablets are typically used as digital design notebooks by using their touch screen as a proxy for paper, along with the user's finger, a capacitive touch pen, or a dedicated stylus as a substitute for a pencil. However, these devices are capable of much more than capturing depictions on "paper." Studies of design processes have shown that the act of design ideation is multimodal in nature: designers sketch on paper, point to or gesture over parts of their sketch, and speak to describe their ideas, raise questions, and evaluate ideas. Capture of designers' actions has typically involved using overhead cameras that can record the sketches as well as the designers' movements, which requires cumbersome equipment. When considering digital interfaces to capture these modes of interaction, stud- 
ies have suggested that encouraging and capturing speech while sketching was useful as it kept visual attention on the sketch, at the same time allowing the designer to convey useful information [14]. Recent developments in personal computing devices have made speech-based interfaces commonplace thanks to digital voice assistants such as Alexa, Cortana, and Siri. Additionally, research directions in HCI have explored the combination of touch and in-air gestures on hand-held devices [9], the use of above-the-surface sensing to anticipate user interactions [21], and the use of 3D input with a custom stylus that enables spatial interactions around a device [46]. Still, these novel technologies have yet to be employed for capturing meta-data and design rationale. Part of the reason for this gap in the literature may be that it is still not clear which meta-data to capture, and how to present it back to the designer to best support the creative process.

In order to exploit the growing sensory capabilities of handheld devices in ways that aid the design process, one needs to first understand designers' actions and underlying intents. In this paper, we address this need by conducting an observational study of 6 experienced practitioners engaged in design ideation tasks. We focus on individual designers rather than teams, as we are interested in what actions of designers can be used to store their ideas. Participants followed a thinkaloud protocol, exploring solutions to a given design problem using a traditional pen-and-paper medium. We video and audio-recorded the participants, and qualitatively coded all occurrences of physical actions: actions that produced marks on paper (such as sketching and annotating), as well as actions that did not (such as pointing and gesturing). We also coded all occurrences of the designer's intent, which referred to what the designer was trying to do: searching for solutions, describing a design solution, or analyzing a design. By identifying co-occurrences between the designer's intent and the physical actions, we determine the actions designers use to express their intent. Based on this intent-to-action mapping, and based on our observations from the study, we then formulate a set of implications for those seeking to create the next generation of digital design notebooks that offer rich interaction modalities.

The contributions of this paper are: (1) a review of research in design methods and digital support for design to identify potential research areas to capture and record designers' intent, (2) a map of designers' actions to designers' intent based on observational studies conducted with design practitioners, and (3) a set of implications for HCI designers and engineers for capturing designers' intent.

\section{BACKGROUND}

Donald Schön, in his paper on the challenges of computer support for design knowledge capture [33], states, "The designer designs not only with the mind, but with the body and senses, a fact that poses an interesting challenge to computers." Yet, studies of designers' behavior has largely focused on uncovering their cognitive actions. While there are a few bodies of work that also emphasize designers' physical behavior, the typical focus is on design methods, and not on digital support. Research on multimodal interactions for design has highlighted the importance of using speech and actions along with sketches, but typically use video and audio to capture these actions. The recent developments in non-traditional interaction techniques that include tangible, haptic, verbal, and even full-body interactions, have created an opportunity to provide computational support for designers by capturing their intent and rationale through their actions, speech, and sketches. In this section, we review work in the fields of design research, HCI support for ideation, and research on multimodal interactions for design.

\section{Sketching in Design Ideation}

Sketching during design ideation typically involves quickly drawn, loosely structured visual representations concerned with broad features rather than detail [27]. Sketches serve as an extension to working memory, reducing the burden of storing and simultaneously operating on content [41]. In such contexts, sketches can convey both, geometric and symbolic information about a designers' idea [28]. The role played by sketches in design ideation can be broadly classified into three themes [43], (1) "thinking sketches," that support cognitive aspects of idea generation, exploration, and evaluation, (2) "talking sketches," that serve as a means for communicating ideas, and (3) "storing sketches," that act as an external memory aid.

Understanding designers' cognitive processes has been an important focus area for previous research in this domain. An early instance of such work is Goldschmidt's [17] analysis of "study sketches"-quick-and-dirty sketches that help the designer reason their way through a design space. In this work, Goldschmidt models the design process as a dialectic process between "seeing as"- - seeing figural properties in a sketch and "seeing that"-eliciting non-figural properties from the sketch. Studies by others $[16,34]$ also suggest that visual display of information through sketching allows reinterpretation, a vital component of design ideation. While these studies discuss designers' physical actions, they do so with the primary motive of mapping them back to their cognitive processes. Suwa et al. [38] extend earlier work by Suwa \& Tversky [39] and create a coding scheme for representing designers' physical and nonphysical actions. While their work provides insights into the types of physical actions used and relationships among them, it does not discuss means for supporting them.

Studies on design ideation indicate that, apart from sketching, designers use a diverse set of verbal and visual strategies for conveying ideas [35], such as pointing and verbal utterances [45]. Digital design notebooks have brought a renewed focus on supporting such physical actions [26], but doing so requires an understanding of both the spatiotemporal properties as well as the intent of the designer's actions.

\section{Digital Support for Design Ideation}

Sutherland's SketchPAD [37] remains the seminal work in interactive design applications, paving the way for ComputerAided Design (CAD) systems and digital sketching interfaces. Since then, the increasing use of digital devices in design ideation has now made it possible to record and share one's voice and physical actions along with sketches. Such actions have been shown to be useful for communication, both with 
other designers and with one's "future self", who may refer to these sketches in the future for inspiration.

Computer support tools for creative design should thus support both individuals and teams [36]. Yet, digital support tools for ideation that goes beyond capturing sketches has typically focused on idea sharing via sketches, such as computermediated group ideation [10,19], team-based approaches to sketching [30,31,47], and immersive environments for collaborative sketching $[13,15]$. Computer support for individual exploration of design spaces has primarily focused on implementing algorithmic approaches to design, such as the Theory of Inventive Problem-Solving (TRIZ) [11], or aiding synthesis through shape grammar and analogy-based design [8].

In addition, observational studies focusing on designer's expression typically focus on the sketching activity. Studies of gestures and other forms of communication are conducted in the context of collaborative work. For instance, a study by Tang [40] on collaborative work found that hand gestures are often used to communicate significant information, and that the process of creating drawings conveys much more information than the drawings themselves. Similarly, an analysis of face-to-face interactions in design teams by Bekker et al. [6] reveals that designers typically use kinetic gestures to enact interactions between users and products, spatial gestures to show distances, sizes, and shapes, and finally pointing gestures to refer to objects/persons/places, to list items, and to contrast two entities. There are fewer instances of computer support tools that emulate a communication between a designer sketching an idea and the same designer interpreting that idea. This notion, called "representational talkback" has been simulated in the form of digital sketching applications that preserve the ambiguity of the sketches [22]. Thus, while studies have shown that the act of sketching and the gestures that accompany it are important, little work on digital support for design ideation focuses on understanding and capturing these forms of expression. Some instances of digital support for multimodal capture of designers' actions are listed in the following section. While most of these systems also simulate collaborative work, they highlight the importance of multimodal capture of designers' actions, and the need for a systematic mapping of actions and intent.

\section{Multimodal Interfaces for Sketching}

Multimodal interfaces for sketching have been researched for over two decades. Speech was considered a natural accompaniment to the sketching action as it occurred over an auditory channel, thus allowing visual attention to remain on the sketch [14]. Gross et al. [18] developed a system they called the design amanuensis that captured and associated co-occurring sketch strokes and speech to be played back together, in an imitation of how design researchers go over design protocol data. Adler et al. [3] also supported speech and sketching as a major aspect of multimodal interfaces for early design, but sought to aid collaboration by having one designer "explain" their sketches while sketching to another designer who may not be physically present, but can play back the explanation when needed. They identified "trimodal" interactions of speech, sketch, and gesture that would be necessary to communicate ideas, and suggested ways in which speech and gestures can be used to both convey additional information over the sketch, and in some instances, modify the sketch to reflect designers' intent [2]. In a later work, Adler [1] uses this approach in a multimodal interactive dialogue system (MIDOS) that takes sketching and speech inputs from the user, uses a physics simulator to interpret the sketched structure, and interactively asks the user to resolve ambiguities. Similarly, Bischel et al. [7] use speech features, specifically (spoken) descriptions of actions, to separate sketch strokes that portray structure from explanatory strokes, such as arrows indicating motion or circles that draw focus to specific parts of the sketch.

While most speech-based sketching technologies use the scenario of collaborative work to capture speech, studies have shown that such technology allows users to replay the audio from previous ideas, and develop variations, which leads to better creative outcomes [32]. The notion of a designer talking by herself or to her device may have been strange in the past, but recent proliferation in digital voice assistants such as Alexa, Siri, and Google Assistant have made the act of speaking to one's devices more commonplace. While most of the work discussed in this section uses cumbersome laboratory setups, this is no longer necessary: the smartphone and tablet, with its capacitive sensors, camera, microphone, inertial measurement unit, and other sensors is increasingly better equipped for multimodal interactions such as the ones described above. Interaction modalities that use touch, grip, and in-air gestures have been suggested for context-sensitive interactions [9,21]. Design and HCI research is thus at a stage where the capability of sensing, understanding, and capturing a designer's intentions based on their actions and context is rapidly being realized. To this end, we intend to understand what a designer expresses in the act of designing, and how they express them, with the goal of providing a set of implications for engineers and researchers seeking to create the next generation of digital design notebooks. Our motivation behind this study is to support HCI researchers who are starting to look at around-the-device interactions [20] as part of the multimodal interaction set, which can be a less intrusive and more intuitive way to capture the designer's actions.

\section{OBSERVATIONAL STUDY}

In order to understand the way designers express their intent, we need to observe them in a real-world problem-solving scenario. Protocol analysis-qualitative analysis of a series of temporal observations - has been used to analyze such behavior [38]. Since we are looking at design problem-solving behavior, we adapted Structural Analysis [5], a systematic approach for analyzing verbal protocols that reveals the subject's problem-solving approaches and thought processes.

For this analysis, we first conducted a observational study using a think-aloud protocol, where the participant is given a design task, and asked to verbalize their thought process. The study was set up to mimic a digital design notebook capable of capturing voice and physical actions in real time in order to associate actions to intent. A microphone and camera (pointed at the notebook) were used to capture audio and physical interactions. The recorded video was qualitatively coded along two main categories: designers' intent, i.e. behaviors 
or utterances that reflected a specific intent pertinent to the design task, and designers' actions, i.e. nonverbal actions performed by the designers during the task. We focused on individual designers as opposed to a team because (a) we are interested in the reflective conversation between the designer and their sketch [4], and (b) our goal is to envision a system where individual designers can use multimodal interactions (including speech) to store their ideas.

\section{Participants}

We recruited six paid participants ( 3 female, 3 male, age 18 40 ), five of whom were Ph.D. students in mechanical engineering, and one a postdoctoral researcher in mechanical engineering with a design major. One participant was left-handed, and all participants had normal or corrected-to-normal vision. We chose experienced participants for this study to lend higher ecological validity to the identified behaviors. Experienced participants would also show lower variability and thus even 6 participants can provide reliable data.

\section{Tasks}

Participants were given one of two design tasks:

T1 Design a bicycle rear suspension such that the horizontal distance between the front and the rear wheels does not vary when the suspension is engaged.

T2 Design a coffee container that allows astronauts to drink hot coffee safely under zero(micro)-gravity conditions.

The two tasks were chosen to observe how designers approach a problem from a familiar domain, and one from an unfamiliar domain. We were also interested in learning if there is a difference in designers' actions when designing in varied domains. T1 was adapted from a case study by Ullman [42], and focused on mechanical design, a domain familiar to the participants, yet challenging enough to require iteration. T2 was chosen to take participants outside their comfort zone with a non-traditional mechanical design problem. The task lasted 20 minutes, during which the participant generated solutions for the given design problem. We asked participants to generate a minimum of three concepts for a given design task, to push their exploration beyond common solutions [25].

Searching for existing designs also forms a primary component of the design process. However, in order to keep the study focused on the act of designing, we discouraged participants from searching for existing designs at the very beginning. However, if they felt they were stuck, or were unclear about the appearance of a component or mechanism, we allowed them to use online search for reference. We also asked participants to follow up their sketching with explanations of their designs ( $\sim 5$ mins). This paper focuses on understanding the designer's thought process during design and not on the designs themselves. We therefore decided not to code the final explanations. However, we did use their gestures during the explanations to verify they were a subset of the gestures they made while designing.

\section{Conditions \& Equipment}

All participants were remotely located, and the tasks were thus conducted via a videoconferencing setup. Participants used webcams pointing down toward their table, to stream their design activity. For the task itself, all participants used white U.S. letter paper $(8.5$ in $\times 11$ in $)$, with pencils, erasers, and markers for sketching. They were audio and video recorded at the researcher's end, and all sketches created by participants were scanned and retained for the study.

\section{Analysis}

In order to identify categories of designers' actions and verbalizations that describe designers' intent, we coded the video recording of the design tasks. The six resulting videos were coded by two of the authors of this paper, with an overlap of one video $(\sim 17 \%)$ between them. To do this, one coder initially created a set of memos for one design task, identifying a set of actions and intent categories. Both coders discussed each category to finalize a codebook. The final set of codes used, their descriptions, and samples from the data are provided in Table 1. Both coders then independently coded the same video. Using five-second windows to compare codes, they reached an agreement Cohen's kappa ( ) of 0.83, $p<0.001$. A sample set of codes (by one coder) for a ten-minute segment of one participant's video is shown in Fig. 2.

In order to identify the actions that accompany a particular intent, we created a coding co-occurrence matrix between the codes categorized under "designer intent" and those under "designer action". For the scope of this study, our interest lies primarily in understanding how different designers may express similar intents. Each cell of our co-occurrence matrix (Table 2) thus reflects the number of participants observed using an action to reflect an intent, rather than the number of co-occurrences coded across participants.

\section{RESULTS \& DISCUSSION}

The results from the protocol analysis exhibit distinct as well as common actions across designers. We discuss the results from our analysis from two perspectives:

- Diversity of Actions: First, our analysis focuses on identifying the diversity in physical actions used to convey design intent. This provides an understanding of the kinds of interactions that future digital design notebooks need to support.

- Intent Expressed: Next, we discuss the use of physical actions during different cognitive episodes (periods of designer behavior that reflect an intent). Understanding such co-occurrences can enable identification of design intent through combinations of utterances, marks on paper and gestures. This in turn can provide vital insights for creating systems that can capture designers' physical actions without intrusion into their cognitive episodes, such as synthesizing a solution or analyzing a design.

\section{Diversity of Physical Actions}

To understand the diversity in physical actions used we explore diversity in (1) physical realization and (2) representation. Both these facets provide vital insights to researchers interested in supporting such physical actions through HCI interfaces. Diversity in physical realization is discussed in Table 3. In this table, we map distinct physical actions to the intent we observed when designers performed these physical 


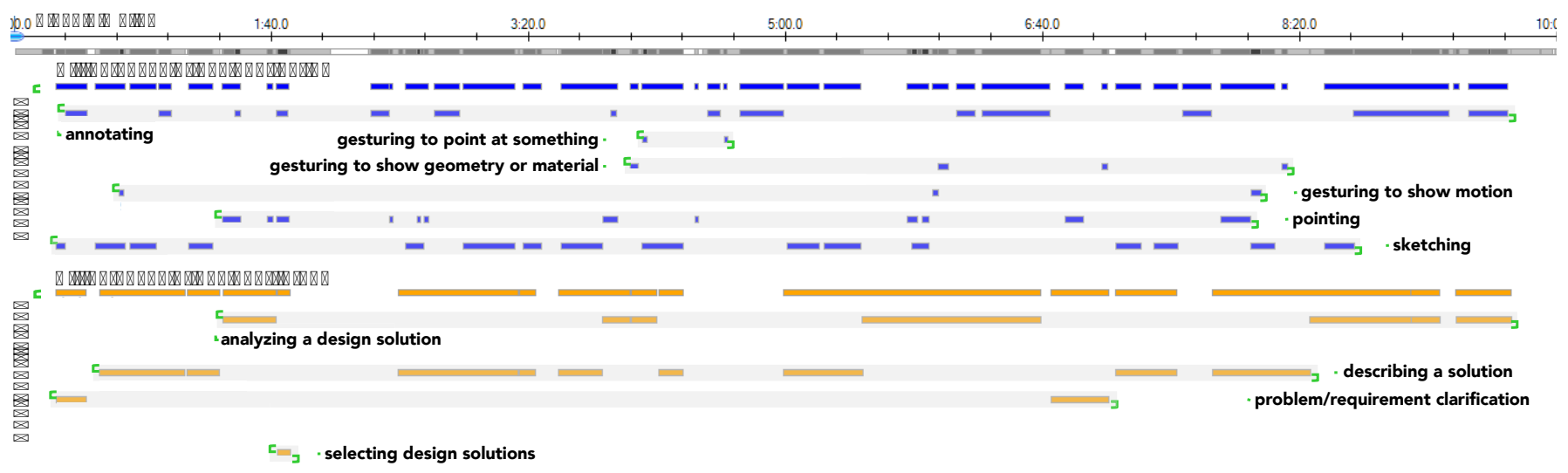

Figure 2. Screen capture of a ten-minute coded segment of one participant video, showing all instances of designer actions (blue) and designer intent (orange). Designer actions such as pointing and gesturing are discrete events that mostly co-occur with, or closely precede/succeed sketching and annotating actions. Coding designer intent based on verbalizations and context helps understand the purpose behind these actions.

\begin{tabular}{|c|c|c|}
\hline Coding Category & Description & Coded Examples \\
\hline \multicolumn{3}{|l|}{ Designer Intent } \\
\hline $\begin{array}{l}\text { Problem or requirement } \\
\text { clarification }\end{array}$ & $\begin{array}{l}\text { Participant expresses their understanding of the design } \\
\text { requirements }\end{array}$ & $\begin{array}{l}\text { "That means somehow we need to attach this wheel with } \\
\text { a suspension system that only moves in this direction" } \\
\text { (gestures) }\end{array}$ \\
\hline Searching for solutions & $\begin{array}{l}\text { Participant searches externally (online/books) for } \\
\text { solutions to the design problem }\end{array}$ & $\begin{array}{l}\text { "I'm searching for" (types on computer as he speaks) } \\
\text { "straight... line... mechanism..., and the first one that } \\
\text { comes up is actually different than what I had seen } \\
\text { before." }\end{array}$ \\
\hline Describing a solution & $\begin{array}{l}\text { The participant describes a solution to the design } \\
\text { problem as they are thinking through the solution }\end{array}$ & $\begin{array}{l}\text { "It should have a temperature sensor in here..." (starts } \\
\text { sketching) "so this one shows the - whether the - } \\
\text { how hot the cup is..." }\end{array}$ \\
\hline Analyzing a design solution & $\begin{array}{l}\text { Participant analyzes a solution to find advantages, } \\
\text { shortcomings, or limitations. }\end{array}$ & $\begin{array}{l}\text { "We could do a... Hamster bottle, um," (starts sketching) } \\
\text { "so it will... oh, but there's zero gravity. So that won't } \\
\text { work." }\end{array}$ \\
\hline Selecting design solutions & $\begin{array}{l}\text { Participant selects potential solutions from a set of } \\
\text { candidates they have generated. }\end{array}$ & $\begin{array}{l}\text { "So, at least one is this, (draws arrow to point at a con- } \\
\text { cept) another is this..." (draws arrow to point at } \\
\text { another concept) }\end{array}$ \\
\hline $\begin{array}{l}\text { Describing a hypothetical design } \\
\text { scenario }\end{array}$ & $\begin{array}{l}\text { Participant discusses a hypothetical scenario in which } \\
\text { s/he is solving the given design problem }\end{array}$ & $\begin{array}{l}\text { "I'll need to go back and ask the customer" (writes note } \\
\text { on sketch) "if this is okay." }\end{array}$ \\
\hline \multicolumn{3}{|l|}{ Designer Action } \\
\hline Sketching & $\begin{array}{l}\text { Participant makes a mark on the paper that represents } \\
\text { form, geometry, locations, or lines of action. }\end{array}$ & $\begin{array}{l}\text { "It also..." (sketches as he talks) "is connected..." } \\
\text { (keeps sketching) "to another piece from the main } \\
\text { chassis..." }\end{array}$ \\
\hline Annotating & $\begin{array}{l}\text { Participant makes marks such as text, numbers, and } \\
\text { symbols. }\end{array}$ & $\begin{array}{l}\text { "... it will continue to go through these small, thin, } \\
\text { tubules" (writes 'tubules' and draws an arrow in- } \\
\text { dicating part of sketch) }\end{array}$ \\
\hline Pointing & $\begin{array}{l}\text { Participant points to one or more parts of the sketch or } \\
\text { specific artifact with one or more fingers(s), pencil(s), } \\
\text { and/or other objects. }\end{array}$ & $\begin{array}{l}\text { "The system between the axle" (points with right in- } \\
\text { dex finger to axle on sketch) "and the pedals" } \\
\text { (points with left index finger to pedals) "is the same } \\
\text { length..." }\end{array}$ \\
\hline Gesturing to point & $\begin{array}{l}\text { Participant indicates a part of a sketch or artifact, but } \\
\text { uses a non-static gesture to point. }\end{array}$ & $\begin{array}{l}\text { "... and that's going to make this mechanism" (makes } \\
\text { circular gesture over the mechanism) "pretty small, } \\
\text { most likely." }\end{array}$ \\
\hline $\begin{array}{l}\text { Gesturing to show } \\
\text { geometry or material }\end{array}$ & $\begin{array}{l}\text { Participant makes a gesture with hand(s) and/or } \\
\text { pencil(s) to show shape, orientation, distance, or } \\
\text { material. }\end{array}$ & $\begin{array}{l}\text { "... and then this hydraulic system kind of working to } \\
\text { maintain this distance (makes back-and-forth move- } \\
\text { ment with pen) here..." }\end{array}$ \\
\hline Gesturing to show motion & $\begin{array}{l}\text { Participant makes a gesture with hand(s) and/or } \\
\text { pencil(s) to show movement of a component or } \\
\text { assembly. }\end{array}$ & $\begin{array}{l}\text { "This will give us our full vertical deformation" (makes } \\
\text { oscillatory motion with pencil over corresponding } \\
\text { part of sketch) }\end{array}$ \\
\hline
\end{tabular}

Table 1. Coding categories, descriptions, and examples

actions. We also further classify each action into "marking" and "non-marking" actions, based on whether the pencil makes a mark on the paper while performing an action.
The significance of the table is apparent when studying the variety of sketches drawn by participants (Fig. 3). Some sketches are heavily annotated, while others are not annotated at all. Similarly, some sketches show a high-fidelity representation of the concept, while others show the concept as an abstract, 


\begin{tabular}{lcccccc}
\hline Designer Intent & Sketching & Annotating & Pointing & $\begin{array}{c}\text { Gesturing } \\
\text { to point }\end{array}$ & $\begin{array}{c}\text { Gesturing to show } \\
\text { geometry/material }\end{array}$ & $\begin{array}{c}\text { Gesturing to } \\
\text { show motion }\end{array}$ \\
\hline Problem/requirement clarification & 4 & 2 & 3 & 1 & 3 & 1 \\
Searching for solutions & 1 & 0 & 1 & 0 & 0 & 0 \\
Describing a solution & 6 & 5 & 6 & 6 & 5 & 5 \\
Analyzing a design solution & 6 & 3 & 4 & 4 & 2 & 0 \\
Describing a design scenario & 0 & 1 & 1 & 0 & 0 & 0 \\
Selecting a design solution & 0 & 1 & 1 & 0 & 0 & 0 \\
\hline
\end{tabular}

Table 2. Number of participants (max: 6) that used an action (columns) to express a particular intent (rows) at least once.

formal mechanism diagram. Table 3 , shows the various ways in which participants expressed their intent. The combinations of physical actions described in the table, provide an understanding of how sketching and non-sketching actions are used to convey intent in design ideation. Table 3 also highlights the extent of marking actions whose meanings are lost without the accompanying verbalizations, and non-marking actions that are lost entirely without a means to record them.

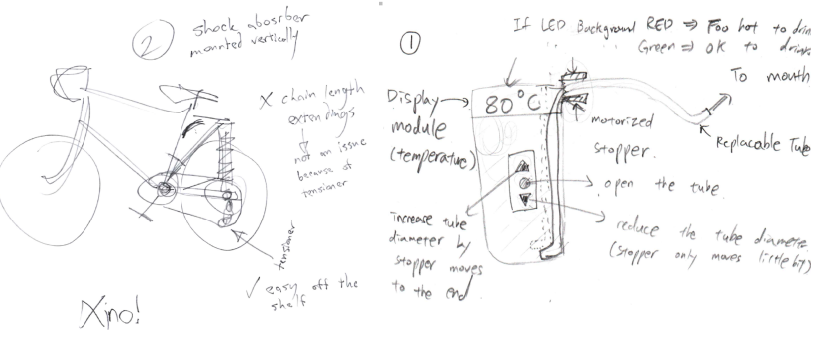

Figure 3. Sample sketches of a bike suspension (Task T1) and a microgravity coffee container (Task T2). The sketches show the variety of abstraction in the sketches, and the variation in their annotations.

These sections below discuss prevalent as well as unique means used for representing each of the coded physical actions. We also highlight the challenges/opportunities they present for creating future digital design notebooks.

Sketching: True to the notion of sketching in design ideation being "quick and dirty," participants did not spend any time on beautifying their concept sketches. We also noticed that participants did not focus on the expressiveness of their strokes. Thick lines and shading were primarily used for emphasizing components that were either thin/small or contained complex geometry, such as when sketching a one-way valve in a mouthpiece, or insulation around the cups. We also noticed that designers used "datum features" (ground, center lines) to reference the spatial location of sketch components. Such features can provide vital clues for inferring implicit intent including relational attributes among sketch components (e.g. A is contained by B), symmetry, and trajectories.

We noticed two kinds of erasing actions for editing marks on the paper: (1) corrections made when the mark was significantly different from what was intended, and (2) modification of drawn geometric features when refining or adding new ideas to the sketch. The latter is particularly important as information about the evolution of ideas is lost by this physical action. This limitation is inherent to paper-based design notebooks. Recent digital design frameworks such as skWiki [47] partly address this issue by storing sketch histories through explicit user interactions. Capturing and representing metadata associated with these actions of erasing and editing can further capture the rationale behind these changes.

Annotating: We also noticed that text annotations were primarily used for explaining mechanisms and interface elements (such as buttons). Thus, they act as a "user manual" that helps resolve ambiguities when such sketches are reused in the future. However, there were significant differences in the usage of sketch annotations between participants. P1 had the highest code coverage for annotation at $29 \%$ (percentage of overall task time spent annotating), while P5 had the lowest (3\%). This represents the total time spent annotating, not the number of instances of annotation observed.

We also observed some variations in the nature and manner of the annotations made. There were instances of "floating annotations," which were not in physical proximity of, or linked to sketch elements. There were also instances of "crossed annotating," or annotations spaced temporally, in which designers paused while annotating one part of a sketch to draw an arrow to a previous annotation. Without observing the designers actually creating these annotations, it would be difficult to unambiguously resolve the information they represent. Other variations in annotating included directional inconsistencies where annotation arrows sometimes pointed at the sketch, and sometimes at the text, and finally occluding annotations that were made on top of sketch elements (see Table 3).

Pointing: Pointing was the second most common action observed, following sketching. This makes sense, given the think-aloud nature of the study: participants would often talk about their sketch and point to components and assemblies to explain what they are doing. The most common mode of behavior observed for pointing was using the pencil or the finger of either hand (see Table 3). We also observed participants using a combination of pencil and a finger, or two fingers, for pointing at two or more portions in the sketch, often to indicate relative positions of components. Sometimes, pointing was also done by creating marks on the paper, such as arrows (without annotations), or marking over a spot on the sketch, typically to refer to a point, axis, or center. An interesting behavior we observed was designers pointing to items that do not exist in the sketch, but are important considerations for the overall idea (e.g. amount of coffee in the cup). In such instances, verbal utterances are the only sources of information that tell us about the object to which the designer refers.

The duration of pointing varied significantly between occurrences as well as participants: the pointing action that lasted the shortest $(0.4 \mathrm{sec})$ occurred when a participant $(\mathrm{P} 1)$ pointed 


\begin{tabular}{|c|c|c|c|c|c|}
\hline \multirow{2}{*}{ Observed Action } & \multirow{2}{*}{ Observed Intent } & \multirow{2}{*}{ Type } & \multicolumn{3}{|c|}{ Spatial Classification } \\
\hline & & & $\begin{array}{l}\text { On the } \\
\text { Surface }\end{array}$ & $\begin{array}{l}\text { Over the } \\
\text { Surface }\end{array}$ & $\begin{array}{l}\text { Away from } \\
\text { the Surface }\end{array}$ \\
\hline \multicolumn{6}{|l|}{ Sketching } \\
\hline Marking strokes to create datum features & $\begin{array}{l}\text { Establishing spatial relationships among } \\
\text { components in sketch }\end{array}$ & Marking & $\checkmark$ & - & - \\
\hline Editing strokes & Refining ideas represented in sketches & Marking & $\checkmark$ & - & - \\
\hline Floating annotations & Linking ideas to non-sketched elements & Marking & $\checkmark$ & - & - \\
\hline Crossed annotations & Refer to previous annotations in sketch & Marking & $\checkmark$ & - & - \\
\hline Occluding annotations & $\begin{array}{l}\text { Clarify components of the sketch using } \\
\text { annotations on top of sketch components }\end{array}$ & Marking & $\checkmark$ & - & - \\
\hline \multicolumn{6}{|l|}{ Pointing } \\
\hline Pointing to sketch with pencil & Refer to sketch and non-sketched elements & Non-marking & - & $\checkmark$ & - \\
\hline Marking on the sketch & Refer to an assembly or an area & Marking & $\checkmark$ & - & - \\
\hline Tracing over the sketch (hover) & Refer to an assembly or an area & Non-marking & - & $\checkmark$ & - \\
\hline $\begin{array}{l}\text { Hand pose \& gestures based on sketched } \\
\text { geometry }\end{array}$ & Design moves [33] & Non-marking & - & $\checkmark$ & - \\
\hline \multicolumn{6}{|l|}{ Gesturing to describe geometry/material } \\
\hline Tracing over the sketch (hover) & Refer to distance, orientation, or material & Non-marking & - & $\checkmark$ & - \\
\hline $\begin{array}{l}\text { Gesture using } 2 \text { fingers (same or different } \\
\text { hand) }\end{array}$ & Refer to distances and orientations & Non-marking & - & $\checkmark$ & $\checkmark$ \\
\hline Gesture using 1 finger and pencil & Refer to distances and orientations & Non-marking & - & $\checkmark$ & $\checkmark$ \\
\hline Hand as a proxy (using a specific pose) & $\begin{array}{l}\text { Proxy for a component or assembly, refer to a } \\
\text { shape }\end{array}$ & Non-marking & - & $\checkmark$ & $\checkmark$ \\
\hline \multicolumn{6}{|l|}{ Gesturing to describe motion } \\
\hline $\begin{array}{l}\text { Tracing over the motion trajectory using } \\
\text { finger(s) on or over the paper }\end{array}$ & Describe a motion of a component & Non-marking & - & $\checkmark$ & - \\
\hline
\end{tabular}

Table 3. Types of coded physical actions observed for P1-P6. Intent was interpreted based on accompanying verbalizations (see Table 1).

to a component while he was sketching it. The pointing action that lasted the longest $(11 \mathrm{sec})$ happened when another participant $(\mathrm{P} 2)$ pointed to a component with his non-dominant hand for reference, and considered the geometrical constraints in the rest of the assembly, using the pencil in his dominant hand to point to the corresponding features. Separating the intent of such simultaneously occurring pointing actions requires access to the sketch and verbalizations at the same time.

This same participant also often pointed to earlier sketches he had made to compare and contrast features with his current design. Such pointing actions indicate that ideas in the older sketch are being used as a reference for the new sketch. If captured, such actions can provide a richer understanding of lateral transformations [16] in design ideation.

Gesturing to Point: There is a subtle difference between "gesturing to point" and "point", in that the intent is similar, but the object and the mechanism are different. Pointing is often a more precise and discrete act: the designer points or jabs with his finger, keeps it stationary for a short while, and moves it away. Gesturing to point, on the other hand is a less precise, and more continuous act: it occurs when referring to a more general object such as an assembly or an area, and the hand/pencil is in motion for the duration of the gesture.

Table 3 shows the various forms of gesturing-to-point observed with the participants. As with pointing, there are marking and non-marking gestures. The marking gestures take the form of drawing over an existing line, feature, or component, to refer to it. We also observed a corresponding non-marking gesture where the participant traced out the rough shape of the line, feature, or component in the air just above the paper. A rougher, more approximate version of this has already been discussed above, where the participant makes a circular motion above the area of interest to indicate an assembly or region (see "Gesturing to point" in Table 1 for an example).

A less-common gesture in this category was the use of the thumb and forefinger spaced out over a component or feature of the sketch. The space between the thumb and forefinger now represented the component of interest, and the participant could then "place" the component at other locations to explore different scenarios or alternatives. These are indicative of "design moves" [33] where the designer explores configuration changes in a design. Capturing such moves not only keeps a 
record of the scenarios considered, but also gives us insight into the designer's creative and analytical processes.

Gesturing to Describe Geometry/Material: Table 3 shows that "gesturing to describe geometry/material" is unique: no version of it produced marks on paper. Main uses of this gesture referred to situations where participants indicated distance, orientation, or in some cases, material. Participant P1, for instance, worked on the coffee container task (T2), and used a rapid series of diagonal strokes above the paper to indicate the amount of coffee (full/half full) he considers to be in the container. Another participant (P5) used a tracing-over gesture in the air to indicate an insulated region in her design.

Participants also used combinations of two fingers (same hand), two fingers (both hands), or pencil and finger (both hands) to indicate distances and orientations above the sketch. This is similar to the "gesturing to point" with the thumb and forefinger discussed above, but the intent here is to describe a property rather than to explore scenarios. Multiple participants also showed distances and orientations using a finger or pencil by rapidly moving it back and forth between two points.

Finally, two participants used their hands as a proxy to indicate the shape of a component. P2 used both hands to indicate the shape of mechanism linkage used in a bicycle design, while P3 shaped her dominant hand to show the geometry of the cup when discussing the need for insulation. These gestures, where the hands are used as a proxy for a component or assembly, are more difficult to track and understand, but for the designer, it is a convenient abstraction to visualize their concept.

Gesturing to Describe Motion: The most common gesture to describe motion was observed to be using the pencil tip over the paper to show the motion trajectory of a component. Some alternatives to this action were the use of a finger instead of the pencil (touching or not touching the paper), and in some cases, showing movement with the pencil tip, but occasionally touching the paper to create a mark.

Multiple fingers were used together on or above the paper to indicate translation, rotation, or deformation. Participant P2 used two fingers touching the paper at two sketched features, and moved them to show a pivoting movement of a bicycle swingarm. P6 used her finger and thumb to indicate mounting points of a spring, and performed a squeezing gesture above the paper with those fingers to indicate spring compression.

Finally, some participants used their hands as proxies while verbally referring to the design to show movements. Participant P5 used both hands to show an opening/closing motion of a lid after sketching it out, to visualize and talk about how it would work, while P3 used her hand to show a squeezing motion to indicate deformation of a coffee container design. There is a significant overlap of gesturing modes with showing geometry and gesturing to point, with the differentiating factor being the intentionality behind the act. This is revealed chiefly through context and verbalizations.

\section{Action vs. Intent: Co-occurrence Cases}

All activities under designer intent were coded based on a combination of verbalizations and observed actions from the video data. Table 2 shows correlations between designer intent and actions through a frequency analysis of co-occurrence among the 6 participants. We noticed that actions were often repeated across the coded intent categories. This reduces their use as a method for discerning between intents. However, the lack of "intent-action" pairs can provide interesting clues about the state of the ideation process. For example, while searching for solutions, designers shifted their focus to the laptop, and therefore very little actions was performed on or above the design notebook. Similarly, activities such as "describing a design scenario" and "selecting a design solution" also seemed to move designers' focus away from the notebook.

From a study of the code coverage, it became apparent that the primary activities reflecting designers' intent during the design tasks were describing solutions (average $44 \%$ of participant time) and analyzing solutions (average $13 \%$ of participant time). Note that "describing a solution" does not just stand for the description of a solution after the fact; it includes the designer thinking through the solution as well. Temporal analysis of the codes shows (Fig. 3) that designers frequently switchedback-and-forth between the two activities. This behavior is indicative of the designer having a dialogue with their design sketch [17]. Table 2 shows individual participant actions for these two primary activities. The table shows that most of the designers' actions occur when they are either describing a solution, or analyzing a solution. This is partly because these two activities dominate the duration of the task, but also because of the reflective dialogue mentioned above. We found that, apart from sketching, the use of physical actions significantly varied across participants for both intents. For example, $\mathrm{P} 6$ had very few $(\mathrm{N}=3)$ annotations actions across the two tasks in comparison to P1 $(\mathrm{N}=37)$. Similarly, even though P2 and P4 worked on the same design problem, the number of pointing-related actions ("pointing" \& "gesturing to point") used by them varies significantly ( $\mathrm{N}=35$ and $\mathrm{N}=6$ respectively). Our analysis of the participants suggests that the use of non-sketching actions in depends on the design problem, designers' habits, and the artifact being created.

To get a better understanding how each action is used in the context of these two activities, we explored episodes of designers' activity in detail. Figure 4 illustrates two such episodes, which were among the most densely-coded periods. Such periods are characterized by multiple physical activities, with designers rapidly switching between them. As discussed earlier, we found that similar actions were repeated across the two intents. We could not observe any specific temporal pattern of actions in these two intents. We also analyzed whether the observed actions made marks-on-paper to understand whether they were captured in the design notebooks used. In the episodes shown in Figure 4, we see that 6/14 coded actions for "describing a sketch" and 6/12 coded actions for "analyzing a sketch" do not create marks on paper. Such actions provide vital clues about decision-making processes in design ideation. To illustrate, consider the following excerpt from P2's analysis, "one problem... no matter how we do this (points to rear wheel)... the reason that bicycles have a bar (points to bicycle pedals)... this is what pivots (gestures to show how it pivots using his hand as a proxy)... and the reason for that is that we 


\begin{tabular}{|c|c|c|c|c|c|c|c|c|c|c|c|c|}
\hline & \multicolumn{6}{|c|}{ Intent: Describing a Solution } & \multicolumn{6}{|c|}{ Intent: Analyzing a Solution } \\
\hline & Sketch & Annotate & Point & $\begin{array}{c}\text { Gesture: } \\
\text { to point }\end{array}$ & $\begin{array}{l}\text { Gesture: } \\
\text { geom./mat.l }\end{array}$ & $\begin{array}{c}\text { Gesture: } \\
\text { motion }\end{array}$ & Sketch & Annotate & Point & $\begin{array}{l}\text { Gesture: } \\
\text { to point }\end{array}$ & $\begin{array}{c}\text { Gesture: } \\
\text { geom./mat.l }\end{array}$ & $\begin{array}{c}\text { Gesture: } \\
\text { motion }\end{array}$ \\
\hline P1 & 26 & 34 & 32 & 5 & 3 & 0 & 4 & 1 & 2 & 3 & 1 & 0 \\
\hline $\mathrm{P} 2^{\star}$ & 22 & 5 & 13 & 3 & 1 & 2 & 6 & 11 & 14 & 5 & 4 & 3 \\
\hline P3 & 13 & 19 & 3 & 2 & 1 & 1 & 1 & 0 & 0 & 0 & 0 & 0 \\
\hline $\mathrm{P} 4^{\star}$ & 24 & 0 & 2 & 4 & 0 & 3 & 2 & 0 & 0 & 0 & 0 & 0 \\
\hline P5 & 12 & 5 & 11 & 2 & 3 & 8 & 2 & 2 & 5 & 3 & 0 & 1 \\
\hline $\mathrm{P} 6^{\star}$ & 14 & 3 & 6 & 6 & 2 & 4 & 1 & 0 & 1 & 1 & 0 & 0 \\
\hline
\end{tabular}

NOTE: Participants marked with $\star$ worked on the bike suspension design task (T1), while the rest worked on the coffee container task (T2)

Table 4. Number of coded instances of designer action for each participant (P1-P6) for the two most-observed instances of designer intent

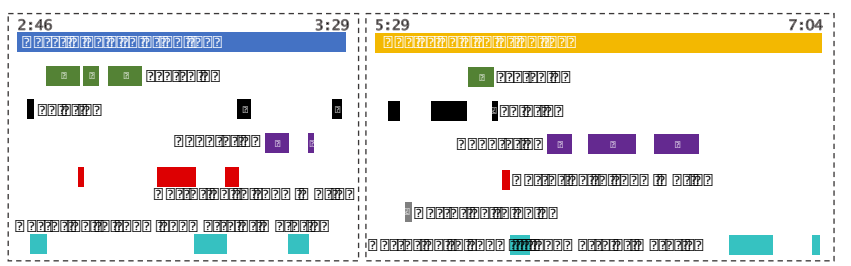

Figure 4. Sample coded segments with time stamps for "describing a solution" and "analyzing a solution". Actions that marked the paper are indicated using boxes with a white vertical line in the center.

do not actually want the distance for the chain to increase." Herein, he highlights vital considerations using a combination of utterances and non-marking gestures. These considerations form the basis for all designs he subsequently creates. Paper design notebooks cannot capture this rationale without the use of accompanying audio and video data.

\section{IMPLICATIONS}

Our results suggest that HCI research on creating digital design notebooks should not treat sketches as the sole focus of design ideation. This focus on sketches has already led to a fixation on creating tools for (1) easing creation, storage, \& sharing of sketches, and (2) enriching sketches through multimedia data such as images, sound, and animation. Results from our analyses show that while designers primarily use sketches and annotations to express themselves, they also use actions such as pointing and gesturing, which, along with verbalizations, could help capture their intent, process, and rationale. Our review of multimodal interfaces for sketching has shown that speech-based and multimodal interfaces are typically designed for collaborative scenarios. With recent developments in voicebased interactions, there is potential for the designer, even when working alone, to verbalize, capture, and contextualize their thought processes. Here we will suggest directions, based on our results, for designing digital design notebooks that capture the designers' intention as well as the designed artifact.

Expanding the Sensory Envelope: Typically, tablet-based sketching interfaces rely on a "sensory envelope" that is limited to the capacitive field over the device surface. Interactions are thus constrained to pen and touch. Typically, existing sketching systems merely capture the digital equivalent of "marks on paper"- -sketches, annotations, and gestures that leave marks on paper, as seen in Table 3. However, they are capable of capturing sketching, annotating, pointing, and gestures that occur just over the surface of the paper. In addition,

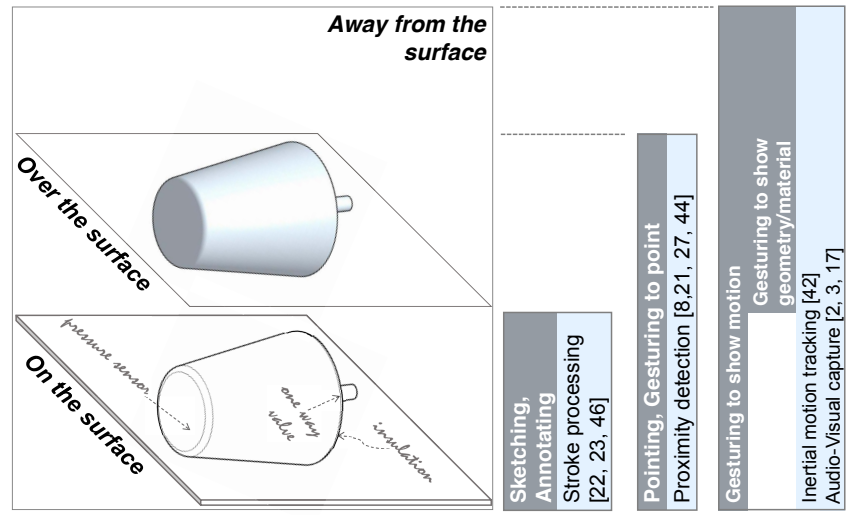

Figure 5. Spatial classification of coded physical actions observed in the studies, and typical research/technological developments that can be used to capture these actions.

we recorded other actions, such as using hands as a proxy for an object, mechanism, or deformation of the design, that occurred "away from the surface." We thus group the observed actions into three categories based on their relation to the sketching surface: on-the-surface, above-the-surface, and away-from-the-surface actions. Interaction modalities such as FingerIO [29] and Expressy [44] are already capable of capturing these and other around-the-device interactions. With the recent proliferation of wearable devices, especially smartwatches, the ability to sense, anticipate, and interpret physical actions has been significantly augmented. For instance, inertial measurement units on smartwatches have been used in combination with touchscreens to interpret the intention behind various touch events [44]. Gesture-based interfaces are becoming more portable thanks to devices like the Leap Motion. Prior work has also used speech to distinguish the intention behind sketch strokes [7]. A similar setup can be used to identify the intention behind on-the-surface and above-thesurface interactions, and through the use of voice recording or transcribed text, associate them with sketched features. Figure 5 maps different on-, over- and away-from-surface actions to related technologies that can be used to capture them.

Capturing Contextual Information: While coding the participant videos to identify designers' intention, it became clear that voice, sketches, annotations, or gestures in isolation cannot provide sufficient information. In fact, it was sometimes necessary to look at a window of time preceding and/or following the episode of interest, in order to establish context. 
While there were dominant actions observed across participants for each identified intent, there was also a fair amount of variations between participants (Table 4). Personal voice assistants such as Alexa and Siri have developed a combination of data mining and machine learning techniques to infer context from a record of user utterances. While such assistants are now built into most handheld devices, issues such as anaphor resolution-identifying what a user refers to when they use a pronoun-still remains an open problem. Augmenting these systems with touch and gesture will, in the context of design ideation, help resolve these issues. For instance, a designer may say while sketching or pointing, "... and then this hydraulic system kind of working to maintain this distance". A synchronized record of speech and pointing actions will resolve what they mean by "this" in each context. Similarly, annotation and sketching processes take longer to complete than accompanying verbalizations, which explains why the verbalizations tend to be spaced out. Keeping a temporal record of verbalizations that co-occurred with the creation-tocompletion time of a sketch can help associate sketches with verbal descriptions, and help identify higher-level processes such as the designer switching between synthesis and analysis modes. Speech, sketch, and gesture have indeed been identified as the "trimodal" interactions of designers [3], as we saw in the background section, early approaches to capture multimodal design interactions use a combination of audio, video, and stroke records to interpret designer intent. This does not stop at capture: researchers envision sketching interfaces for design to be able to recognize, capture, and support the kinds of visual reasoning that designers perform [12]. In our study, our mapping of action, mode, and intent (Table 3 ) was possible only through the accompanying speech (see Table 1). Multimodal interaction systems that aim to capture design intent will thus need to be able to "understand" the designers' speech and use accompanying actions to establish context.

Representing Metadata: For our study, we used video recordings of participants, and qualitative analysis software to identify and code actions and intent. However, playing back relevant video for a sketch is cumbersome and timeconsuming in a design scenario. On-the-surface and abovethe-surface gestures can be represented as animated traces on top of sketches. Gestures to indicate motion can use similar traces, or be used to animate parts of sketches, similar to tools such as Draco [24] and Kitty [23], though inferring relevant sketch segmentation could be a challenge. As mentioned earlier, verbalizations are critical to establish context, and a combination of sketch clustering techniques (e.g. Zhao et al. [48]) and voice annotation can be used to provide relevant data at different levels of resolution.

\section{LIMITATIONS}

While our study helped understand the actions through which designers express their intent, it has a few associated limitations. Firstly, it may be argued that the think-aloud protocol is not indicative of a designer's actual process (they would not talk aloud when designing by themselves). A limitation of using a think-aloud study is that in episodes with prolonged silence, we were unable to infer intent. In a couple of instances, the researcher reminded the designer to verbalize his/her in- tent, which may have affected their thought process. However, there are few alternatives to gain an insight into the designer's thought process. As we saw in the results, verbalizations, while not sufficient, are necessary to obtain context and rationale for a particular design decision. It can also be argued that if the goal is to suggest interactions that can store rich metadata, then speech needs to be one of them. This may not me a great hurdle considering the average users' increasing comfort with digital voice assistants.

Secondly, the design tasks themselves are focused on (1) conceptual design, and (2) come from the domain of physical products. However, the overlap in the types of actions and intents we observed (across the six participants) indicates that they represent common, consistent patterns. We do acknowledge that a follow-up study with a more comprehensive set of design tasks, spanning multiple domains such as interaction design, industrial design, and graphic design, will need to be considered to identify a more diverse set of actions.

Finally, the qualitative coding was more detailed when it came to actions, and less so when it came to designers' intent. For instance, "describing a design solution" can further be divided into stages such as "exploring alternatives", "modifying existing designs", and "creating a new design" etc. However, the focus of the study was on designers' actions reflecting their intent, and less on the design process. We believe that the scope and the depth of the study was sufficient to glean useful insights from the participants' actions.

Paper-based studies such as ours also have the limitation that they do not anticipate new forms of interactions, such as using the device itself as a proxy (to show movement, orientation etc.) for the sketch it contains. However, our goal with this study is to develop a better understanding of how designers express themselves through a medium with which they are familiar and comfortable. Future iterations of this study can accommodate how designers develop new ways to express themselves with digital media.

\section{CONCLUSION}

We have presented results from an observational study of designers engaged in design tasks. Our goal was to understand the role of both sketching and non-sketching actions in expressing the designer's intent. To do this, we categorized the physical actions of the designers under sketching, annotating, pointing, and various gesturing actions, and based on these actions and their verbal utterances, identified their intent. Through our analysis, we understood the importance of preserving "non-marking" actions - actions that do not create a mark on paper-and preserving the context of "marking" actions, in capturing the reasoning behind the designer's actions. Finally, we discuss implications for recording and representing designers' actions in order to capture designers' intent in the next generation of digital design notebooks.

\section{ACKNOWLEDGMENTS}

This work is supported by the U.S. National Science Foundation under grant IIS-1422341. Any opinions, findings, and conclusions expressed in this material are those of the authors and do not necessarily reflect the views of the sponsors. 


\section{REFERENCES}

1. Aaron Adler. 2010. Reasoning About Sketches Using Context, Domain Knowledge, and Interaction with the User. In Proceedings of the AAAI Conference on Visual Representations and Reasoning. 2-5.

2. Aaron Adler and Randall Davis. 2007. Speech and Sketching for Multimodal Design. In ACM SIGGRAPH Courses. DOI : http://dx.doi.org/10.1145/1281500.1281525

3. Aaron Adler, Jacob Eisenstein, Michael Oltmans, Lisa Guttentag, and Randall Davis. 2004. Building the design studio of the future. In AAAI Symposium on Making Pen-Based Interaction Intelligent and Natural. 1-7.

4. Geeta Arjun and Jim Plume. 2006. Collaborative architectural design as a reflective conversation: an agent facilitated system to support collaborative conceptual design. In International Conference of the Arab Society for $C A D$.

5. Wayne A. Bailey and Edwin J. Kay. 1986. Structural Analysis of Verbal Data. ACM SIGCHI Bulletin 18, 4 (1986), 297-301. DOI : http://dx.doi .org/10.1145/1165387.275646

6. Mathilde M. Bekker, Judith S. Olson, and Gary M. Olson. 1995. Analysis of Gestures in Face-to-face Design Teams Provides Guidance for How to Use Groupware in Design. In Proceedings of the ACM Conference on Designing Interactive Systems. 157-166. DOI : http://dx.doi .org/10.1145/225434.225452

7. David Tyler Bischel, Thomas F Stahovich, Eric Jeffrey Peterson, Randall Davis, and Aaron Adler. 2009. Combining Speech and Sketch to Interpret Unconstrained Descriptions of Mechanical Devices.. In Proceedings of the International Jont Conference on Artifical Intelligence, Vol. 9. 1401-1406.

8. Amaresh Chakrabarti, Kristina Shea, Robert Stone, Jonathan Cagan, Matthew Campbell, Noe Vargas Hernandez, and Kristin L Wood. 2011. Computer-based design synthesis research: an overview. Journal of Computing and Information Science in Engineering 11, 2 (2011), 021003.

9. Xiang 'Anthony' Chen, Julia Schwarz, Chris Harrison, Jennifer Mankoff, and Scott E. Hudson. 2014. Air+Touch: Interweaving Touch \& In-air Gestures. In Proceedings of the ACM Symposium on User Interface Software and Technology. 519-525. DOI :

http://dx.doi.org/10.1145/2642918.2647392

10. Andrew Clayphan, Anthony Collins, Christopher Ackad, Bob Kummerfeld, and Judy Kay. 2011. Firestorm: A Brainstorming Application for Collaborative Group Work at Tabletops. In Proceedings of the ACM Conference on Interactive Tabletops and Surfaces. 162-171. DOI : http://dx.doi.org/10.1145/2076354.2076386

11. Marco Aurélio de Carvalho, Jônathas Gobbi Benazi Grillo, and Rodolfo Krul Tessari. 2015. Methodology and
Software for New Product Ideation. Procedia

Engineering 131 (2015), 352 - 358. DOI :

http://dx.doi.org/10.1016/j .proeng. 2015.12.414

12. Ellen Yi-Luen Do. 2002. Drawing marks, acts, and reacts: Toward a computational sketching interface for architectural design. Artificial Intelligence for Engineering Design, Analysis, and Manufacturing 16, 3 (2002), 149-171.

13. Tomás Dorta. 2007. Implementing and assessing the hybrid ideation space: a cognitive artefact for conceptual design. Moon 61 (2007), 77.

14. Kenneth D Forbus, Ronald W Ferguson, and Jeffery M Usher. 2001. Towards a computational model of sketching. In Proceedings of the ACM conference on Intelligent user interfaces. 77-83. DOI : http://dx.doi.org/10.1145/359784.360278

15. Florian Geyer, Jochen Budzinski, and Harald Reiterer. 2012. IdeaVis: A Hybrid Workspace and Interactive Visualization for Paper-based Collaborative Sketching Sessions. In Proceedings of the ACM Nordic Conference on Human-Computer Interaction. 331-340. DOI : http://dx.doi.org/10.1145/2399016.2399069

16. Vinod Goel. 1995. Sketches of thought. MIT Press, Cambridge MA.

17. Gabriela Goldschmidt. 1991. The dialectics of sketching. Creativity Research Journal 4, 2 (1991), 123-143. DOI: http://dx.doi.org/10.1080/10400419109534381

18. Mark D Gross, Ellen Yi-Luen Do, and Brian R Johnson. 2001. The Design Amanuensis: An instrument for multimodal design capture and playback. In Computer Aided Architectural Design Futures, Vol. 1. 1-13.

19. Joshua Hailpern, Erik Hinterbichler, Caryn Leppert, Damon Cook, and Brian P. Bailey. 2007. TEAM STORM: Demonstrating an Interaction Model for Working with Multiple Ideas During Creative Group Work. In Proceedings of the ACM Conference on Creativity $\mathcal{E}$ Cognition. 193-202. DOI: http://dx.doi.org/10.1145/1254960.1254987

20. Chris Harrison. 2010. Appropriated Interaction Surfaces. Computer 43, 6 (2010), 86-89.

21. Ken Hinckley, Seongkook Heo, Michel Pahud, Christian Holz, Hrvoje Benko, Abigail Sellen, Richard Banks, Kenton O'Hara, Gavin Smyth, and William Buxton. 2016. Pre-Touch Sensing for Mobile Interaction. In Proceedings of the ACM Conference on Human Factors in Computing Systems. 2869-2881. DOI : http://dx.doi.org/10.1145/2858036.2858095

22. A. Hoeben and P.J. Stappers. 2005. Direct talkback in computer supported tools for the conceptual stage of design. Knowledge-Based Systems 18, 8 (2005), 407-413. DOI : http://dx.doi.org/10.1016/j.knosys.2005.07.003 Computational Approaches for Early Stages of Design. 
23. Rubaiat Habib Kazi, Fanny Chevalier, Tovi Grossman, and George Fitzmaurice. 2014a. Kitty: Sketching Dynamic and Interactive Illustrations. In Proceedings of the ACM Symposium on User Interface Software and Technology. 395-405. DOI : http://dx.doi.org/10.1145/2642918.2647375

24. Rubaiat Habib Kazi, Fanny Chevalier, Tovi Grossman, Shengdong Zhao, and George Fitzmaurice. 2014b. Draco: Bringing Life to Illustrations with Kinetic Textures. In Proceedings of the ACM Conference on Human Factors in Computing Systems. 351-360. DOI:

http://dx.doi .org/10.1145/2556288.2556987

25. Barry Kudrowitz and Caitlin Dippo. 2013. Getting to the Novel Ideas: Exploring the Alternative Uses Test of Divergent Thinking. In Proceedings of the ASME International Design Engineering Technical Conferences and Computers and Information in Engineering Conference. V005T06A013. D0I: http://dx.doi.org/10.1115/DETC2013-13262

26. Guang Li, Xiang Cao, Sergio Paolantonio, and Feng Tian. 2012. SketchComm: A Tool to Support Rich and Flexible Asynchronous Communication of Early Design Ideas. In Proceedings of the ACM Conference on Computer Supported Cooperative Work. 359-368. DOI : http://dx.doi.org/10.1145/2145204.2145261

27. Robert McKim. 1972. Experiences in visual thinking. (1972).

28. Alexandre Menezes and Bryan Lawson. 2006. How designers perceive sketches. Design Studies 27, 5 (2006), 571-585. DOI : http://dx.doi.org/10.1016/j.destud.2006.02.001

29. Rajalakshmi Nandakumar, Vikram Iyer, Desney Tan, and Shyamnath Gollakota. 2016. FingerIO: Using active sonar for fine-grained finger tracking. In Proceedings of the ACM Conference on Human Factors in Computing Systems. 1515-1525. DOI:

http://dx.doi.org/10.1145/2858036.2858580

30. Cecil Piya, Vinayak, Senthil Chandrasegaran, Niklas Elmqvist, and Karthik Ramani. 2017. Co-3Deator: A Team-First Collaborative 3D Design Ideation Tool. In Proceedings of the ACM Conference on Human Factors in Computing Systems. 6581-6592. DOI : http://dx.doi.org/10.1145/3025453.3025825

31. Ugo Braga Sangiorgi, François Beuvens, and Jean Vanderdonckt. 2012. User Interface Design by Collaborative Sketching. In Proceedings of the Designing Interactive Systems Conference. 378-387. D0I : http://dx.doi.org/10.1145/2317956.2318013

32. Linda C Schmidt, Noe Vargas Hernandez, and Ashley L Ruocco. 2012. Research on encouraging sketching in engineering design. Artificial Intelligence for Engineering Design, Analysis, and Manufacturing 26, 3 (2012), 303-315. DOI :

http://dx.doi.org/10.1017/S0890060412000169

33. Donald A Schön. 1992. Designing as reflective conversation with the materials of a design situation.
Knowledge-based systems 5, 1 (1992), 3-14. DOI : http://dx.doi.org/10.1016/0950-7051(92)90020-G

34. Donald A Schön and Glenn Wiggins. 1992. Kinds of seeing and their functions in designing. Design studies 13, 2 (1992), 135-156. DOI : http://dx.doi.org/10.1016/0142-694X(92)90268-F

35. Stephen Scrivener and Sean Clark. 1994. Sketching in collaborative design. In Interacting With Virtual Environments, Lindsay W. MacDonald and John Vince (Eds.). John Wiley \& Sons, New York, NY.

36. Ben Shneiderman, Gerhard Fischer, Mary Czerwinski, Mitch Resnick, Brad Myers, Linda Candy, Ernest Edmonds, Mike Eisenberg, Elisa Giaccardi, Tom Hewett, Pamela Jennings, Bill Kules, Kumiyo Nakakoji, Jay Nunamaker, Randy Pausch, Ted Selker, Elisabeth Sylvan, and Michael Terry. 2006. Creativity Support Tools: Report From a U.S. National Science Foundation Sponsored Workshop. International Journal of Human-Computer Interaction 20, 2 (2006), 61-77. DOI : http://dx.doi.org/10.1207/s15327590ijhc2002_1

37. Ivan E Sutherland. 1964. Sketchpad a man-machine graphical communication system. Transactions of the Society for Computer Simulation 2, 5 (1964), R-3.

38. Masaki Suwa, Terry Purcell, and John Gero. 1998. Macroscopic analysis of design processes based on a scheme for coding designers' cognitive actions. Design studies 19, 4 (1998), 455-483. DOI :

http://dx.doi.org/10.1016/S0142-694X(98)00016-7

39. Masaki Suwa and Barbara Tversky. 1996. What Architects See in Their Sketches: Implications for Design Tools. In ACM Conference Companion on Human Factors in Computing Systems. 191-192. DOI : http://dx.doi.org/10.1145/257089.257255

40. John C. Tang. 1991. Findings from observational studies of collaborative work. International Journal of Man-Machine Studies 34, 2 (1991), 143-160. DOI : http://dx.doi .org/10.1016/0020-7373(91)90039-A

41. Barbara Tversky. 2002. What do sketches say about thinking. In AAAI Spring Symposium on Sketch Understanding, T. Stahovic, J. Landay, and R. Davis (Eds.). 148-151.

42. David G Ullman. 2009. The Mechanical Design Process. Vol. 4. McGraw-Hill, New York.

43. Remko Van der Lugt. 2005. How sketching can affect the idea generation process in design group meetings. Design studies 26, 2 (2005), 101-122. DOI:

http://dx.doi.org/10.1016/j.destud.2004.08.003

44. Gerard Wilkinson, Ahmed Kharrufa, Jonathan Hook, Bradley Pursglove, Gavin Wood, Hendrik Haeuser, Nils Y. Hammerla, Steve Hodges, and Patrick Olivier. 2016. Expressy: Using a Wrist-worn Inertial Measurement Unit to Add Expressiveness to Touch-based Interactions. In Proceedings of the ACM Conference on Human Factors in Computing Systems. 2832-2844. DOI: http://dx.doi.org/10.1145/2858036.2858223 
45. Anthony Williams and Robert Cowdroy. 2002. How designers communicate ideas to each other in design meetings. In DS 30: Proceedings of DESIGN 2002, the 7th International Design Conference, Dubrovnik.

46. Sang Ho Yoon, Ke Huo, and Karthik Ramani. 2016. TMotion: Embedded 3D Mobile Input Using Magnetic Sensing Technique. In Proceedings of the ACM Conference on Tangible, Embedded, and Embodied Interaction. 21-29. DOI :

http://dx.doi.org/10.1145/2839462.2839463
47. Zhenpeng Zhao, Sriram Karthik Badam, Senthil Chandrasegaran, Deok Gun Park, Niklas Elmqvist, Lorraine Kisselburgh, and Karthik Ramani. 2014. skWiki: A Multimedia Sketching System for Collaborative Creativity. In Proceedings of the ACM Conference on Human Factors in Computing Systems. 1235-1244. DOI : http://dx.doi.org/10.1145/2556288.2557394

48. Zhenpeng Zhao, William Benjamin, Niklas Elmqvist, and Karthik Ramani. 2015. Sketcholution: Interaction histories for sketching. International Journal of Human-Computer Studies 82 (2015), 11 - 20. DOI : http://dx.doi.org/10.1016/j.ijhcs.2015.04.003 